\title{
The occurence of interstellar meteoroids in the vicinity of the Earth
}

\author{
Mária Hajduková Jr. \\ Astronomical Institute of the Slovak Academy of Sciences \\ Dúbravská cesta 9, 84505 Bratislava, The Slovak Republic \\ email: astromia@savba.sk
}

\begin{abstract}
If interstellar meteors are present among the registered meteor orbits, the distribution of the excesses of their heliocentric velocities should correspond to the distribution of radial velocities of close stars. Hence, for the velocity $v_{i}=20 \mathrm{kms}^{-1}$ of an interstellar meteor (with respect to the Sun) we obtain a heliocentric velocity $v_{H}=46.6 \mathrm{kms}^{-1}$ of an interstellar meteor arriving at the Earth. Moreover, a concentration of radiants to the Sun's apex should be observed. An analysis of the hyperbolic meteors among the 4581 photographic orbits of the IAU Meteor Data Center showed that the identification of the vast majority of the hyperbolic orbits in these catalogues has been caused by an erroneous determination of their heliocentric velocity and/or other parameters. Any error in the determination of $v_{H}$, especially near the parabolic limit, can create an artificial hyperbolic orbit that does not really exist. On the basis of photographic meteors from the IAU MDC, the proportion of possible interstellar meteors decreased significantly (greater than 1 order of magnitude) after error analysis and does not exceed the value $2.5 \times 10^{-4}$. Neither any concentration of radiants to the Sun's apex, nor any distribution following the motion of interstellar material has been found.
\end{abstract}

Keywords. Meteors, meteoroids, interplanetary medium

\section{Introduction}

From the position of the whole Solar System in the Galaxy and the studies of processes in the interstellar medium, it is clear that the Solar System is not an isolated system. Its interaction with the interstellar medium should lead to the presence of interstellar particles.

The present work is based on the analysis of 4581 meteor orbits collected in the photographic catalogues of the IAU Meteor Data Center (MDC) (Lindblad et al. 2005). Among them, 527 orbits $(11.5 \%)$ are hyperbolic. It was shown (Hajduková 2008) that the vast majority of hyperbolic orbits has been caused by the erroneous determination of their heliocentric velocity or other parameters and that the number of hyperbolic meteors in the investigated catalogues of MDC does not represent in any case the number of interstellar meteors in observational data. Earlier (Hajduková 1994), from an analysis of the 2910 photographic orbits from the older version of the MDC, it was shown that there is a lack of statistical argument for the presence of real hyperbolic orbits; hence, it is necessary to analyse all special cases individually. This paper deals with the individual approach to the photographic orbits showing extremely high hyperbolic excesses found in 6 of 17 catalogues.

\section{Interstellar meteoroids and meteoroids with hyperbolic orbits}

Meteoroids entering the atmosphere of the Earth and coming from the interstellar medium have heliocentric velocities $v_{H}>v_{p}$, where $v_{p}$ is the parabolic velocity with 
respect to the Sun, and the hyperbolicity of their orbits (with semimajor axis $a<0$ and eccentricity $e>1$ ) is not caused by planetary perturbations inside the Solar System. The difference between hyperbolic and parabolic heliocentric velocity is the hyperbolic excess of heliocentric velocity defined as $\Delta v_{H}=v_{0}\left[(2 / r-1 / a)^{1 / 2}-(2 / r)^{1 / 2}\right]$, where $a$ is measured in $\mathrm{AU}$ and $v_{H}$ in $\mathrm{kms}^{-1} ; v_{0}$ is the mean heliocentric velocity of the Earth, $29.8 \mathrm{kms}^{-1}$ and $r=1$. For this approximation we have selected and analysed individual meteors from the photographic catalogues of the IAU MDC.

The value of semimajor axis, $a$, is very sensitive to the value of the heliocentric velocity $v_{H}$, especially near the parabolic limit. Hence, any error in the determination of $v_{H}$ can create an artificial hyperbolic population that does not really exist. If interstellar meteors are present among the hyperbolic orbits, the distribution of the hyperbolic excesses of their heliocentric velocities should correspond to the distribution of radial velocities of close stars. For the velocity of an interstellar meteor $v_{i}=20 \mathrm{kms}^{-1}$, which represent the relative velocity of the Sun in the nearby stellar environment and taking into account the equation $v_{i}^{2}=v_{H}^{2}-v_{p}^{2}$, with $v_{p}=42.1 \mathrm{kms}^{-1}$, we obtain a heliocentric velocity $v_{H}=46.6 \mathrm{kms}^{-1}$ of an interstellar meteor arriving at the Earth.

\section{Extreme hyperbolic orbits in the IAU MDC}

There are 527 formally hyperbolic orbits (with eccentricity $e>1$ ) among the total of 4581 photographic meteoroid orbits in the MDC catalogues. However, almost half of them (247 orbits) have been identified with the major meteor showers. We have found 59 orbits with $e>1$ and $v_{H}>46.6 \mathrm{kms}^{-1}$ in the photographic catalogues of MDC, of which 54 are from the Dushanbe, Odessa and Kiev catalogues, 3 from the European Fireball Network, 1 from the Nippon Meteor Society and 1 in the McCrosky - Shao catalogue. There is no meteoroid with $v_{H}>46.6 \mathrm{kms}^{-1}$ in the 11 other photographic catalogues, containing more than $2 / 3$ of all photographic orbits. In the most precise 413 Superschmidt orbits from the catalogue of Jacchia and Whipple the largest value of $\Delta v_{H}$ is $0.7 \mathrm{kms}^{-1}$. The presence of $23-26$ shower meteors among this sample of 59 extremely hyperbolic meteors points to very large observational or measurement errors.

From this analysis it can be concluded that many apparent hyperbolic orbits presented in the low quality data are not true hyperbolic meteors. In more precise data, there may be an argument for the presence of true hyperbolic orbits. However, the hyperbolic excesses of the velocities in these cases are very low, about one order less than required from the velocity distribution of neighbouring stars, which argues against the presence of interstellar meteors even in the most precise data. Summarizing the above data, the proportion of real hyperbolic meteors decreased significantly after the error analysis, from $11.5 \times 10^{-2}$ to $2.5 \times 10^{-4}$ of the total number of photographic meteors in the database. Hence, the flux of interstellar meteors is much lower than was declared by the authors of the catalogues, or was believed in some analyses of these observations. It is at least 1 order of magnitude less.

\section{Acknowledgements}

This work was supported by the Slovak Scientific Grant Agency VEGA, grant No 3067.

\section{References}

Lindblad, B. A., Neslušan, L., Porubčan, V., \& Svoreň, J. 2005, Earth, Moon, Planets, 93, 249 Hajduková, M. Jr. 1994, A\&̊A, 288, 330

Hajduková, M. Jr. 2008, Earth, Moon, Planets, 102, 67 